\title{
ON THE HYPERBOLICITY CONSTANT OF CIRCULAR-ARC GRAPHS
}

\author{
ROSALÍO REYES, JOSÉ M. RODRÍGUEZ(1)(2), JOSÉ M. SIGARRETA(1)(2), AND MARÍA VILLETA ${ }^{(1)}$
}

\begin{abstract}
Gromov hyperbolicity is an interesting geometric property, and so it is natural to study it in the context of geometric graphs. It measures the tree-likeness of a graph from a metric viewpoint. In particular, we are interested in circular-arc graphs, which is an important class of geometric intersection graphs. In this paper we give sharp bounds for the hyperbolicity constant of (finite and infinite) circular-arc graphs. Moreover, we obtain bounds for the hyperbolicity constant of the complement and line of any circular-arc graph. In order to do that, we obtain new results about regular, chordal and line graphs which are interesting by themselves.
\end{abstract}

Keywords: Circular graphs; Circular-arc graphs; Geometric graphs; Gromov hyperbolicity; Geodesics. AMS Subject Classification numbers 2010: 05C62; 05C63; 05C10; 05C75; $05 \mathrm{C} 12$.

\section{INTRODUCTION}

The concept of Gromov hyperbolicity grasps the essence of negatively curved spaces like the classical hyperbolic space, simply connected Riemannian manifolds of negative sectional curvature bounded away from 0, and of discrete spaces like trees and the Cayley graphs of many finitely generated groups (see the precise definition of hyperbolicity in the next section). It is remarkable that a simple concept leads to such a rich general theory (see [22, 23]). The first works on Gromov hyperbolic spaces deal with finitely generated groups (see 23]). Initially, Gromov hyperbolic spaces were applied to the study of automatic groups in the science of computation (see, e.g., [37]); indeed, hyperbolic groups are strongly geodesically automatic, i.e., there is an automatic structure on the group [13. This concept appears also in algorithms and networking. For example, it has been shown empirically in 46 that the internet topology embeds with better accuracy into a hyperbolic space than into an Euclidean space of comparable dimension (formal proofs that the distortion is related to the hyperbolicity can be found in [49]); the same holds for many complex networks, see 30. A few algorithmic problems in hyperbolic spaces and hyperbolic graphs have been considered in recent papers (see [29] and the references therein). Furthermore, hyperbolic spaces are useful in secure transmission of information on the network (see [26]).

In [48] it was proved the equivalence of the hyperbolicity of many negatively curved surfaces and the hyperbolicity of a graph related to it; hence, it is useful to know hyperbolicity criteria for graphs from a geometrical viewpoint. In recent years, the study of mathematical properties of Gromov hyperbolic spaces has become a topic of increasing interest in graph theory and its applications (see, e.g., 3, 7, 9, 15, 16, 17, 18, 20, 21, 26, 28, 30, 32, 42, 43, 44, 47, 48, 50, and the references therein).

For a finite graph with $n$ vertices it is possible to compute $\delta(G)$ in time $O\left(n^{3.69}\right)$ [20] (this is improved in 16, 17). Given a Cayley graph (of a presentation with solvable word problem) there is an algorithm which allows to decide if it is hyperbolic [39. A refinement of this approach has been proposed in [12, that allows to do the same for many graphs: in particular, it provides a simple constant-factor approximation of the hyperbolicity constant of a graph on $n$ vertices in $O\left(n^{2}\right)$ time when the graph is given by its distancematrix. However, deciding whether or not a general infinite graph is hyperbolic is usually very difficult.

Date: April 7, 2020.

(1) Supported in part by three grants from Ministerio de Economía y Competititvidad, Agencia Estatal de Investigación (AEI) and Fondo Europeo de Desarrollo Regional (FEDER) (MTM2013-46374-P, MTM2016-78227-C2-1-P and MTM201569323-REDT), Spain.

(2) Supported in part by a grant from CONACYT (FOMIX-CONACyT-UAGro 249818), México. 
Therefore, it is interesting to relate hyperbolicity with other properties of graphs. The papers [8, 9, 50] prove that chordal and $k$-chordal graphs are hyperbolic. These results relating chordality and hyperbolicity are improved in 2, 32]. Some other authors have obtained results on hyperbolicity for particular classes of graphs: vertex-symmetric graphs, bipartite and intersection graphs, bridged graphs, expanders, and interval graphs [10, 18, 28, 31, 40]. In the recent paper [19] new symmetric graph classes are proved to be nonhyperbolic.

A geometric graph is a graph in which the vertices or edges are associated with geometric objects. One of the main classes of geometric graphs are intersection graphs. An intersection graph is a graph in which each vertex is associated with a set and in which vertices are connected by edges whenever the corresponding sets have a nonempty intersection. Since hyperbolicity is an interesting geometric property, it is natural to study this property for some classes of geometric graphs. Intersection graphs are very important in both theoretical and application point of view (see, e.g., 34]). In [40] the authors study the hyperbolicity constant of interval graphs (an interval graph is the intersection graph of a family of intervals on the real line). In this paper we work with circular-arc graphs (another important class of intersection graphs).

A circular-arc graph (or circular graph) is the intersection graph of a family of arcs on the unit circle $S S$. It has one vertex for each arc in the family, and an edge between every pair of corresponding vertices to arcs that intersect. Circular-arc graphs are useful in modeling periodic resource allocation problems in operations research (each arc represents a request for a resource for a specific period repeated in time). They also have applications in different fields such as genetic research, traffic control, computer compiler design and statistics (see, e.g., 38). In [33 appears an $O(n+m)$ time algorithm for recognizing a circular-arc graph (with $n$ vertices and $m$ edges).

Of course, every interval graph can be viewed as a circular-arc graph; if a representation of a circulararc graph $G$ leaves some point of the unit circle uncovered, it is topologically the same as an interval representation of $G$ (by cutting the circle and straighten it out to a straight line); we will use this identification along the paper.

In this paper we give sharp bounds for the hyperbolicity constant of circular-arc graphs (see Theorem 3.1). These bounds are improved in Theorem 3.2 for proper circular-arc graphs. Theorem 3.5 gives a sufficient condition in order to attain the lower bound of $\delta(G)$ in Theorem 3.1; in particular, it shows that this bound is sharp. Propositions 3.3 and 3.4 characterize the circular-arc graphs with small hyperbolicity constant. In Section 4, we obtain bounds in Theorems 4.5 and 4.15 for the hyperbolicity constant of the complement and line of a circular-arc graph, respectively. These theorems improve, for circular-arc graphs, the general bounds for the hyperbolicity constant of the complement and line graphs. In order to do that, we obtain new results about regular, chordal and line graphs which are interesting by themselves (see Theorems 4.3 and 4.14). Although some results of this paper can be viewed as generalizations to the context of circular-arc graphs of results for interval graphs (see [40]), we want to remark that Theorem 4.15 is new even for interval graphs.

Besides [40, in [18] sharp inequalities for the hyperbolicity constant of several classes of intersection graphs are provided. In particular, for line, clique and biclique graphs, and some extensions of line graphs (incidence, total, middle and $k$-edge graphs). Hence, the results in [18] are different from the results in this paper. Furthermore, the bounds in [18] refer to the hyperbolicity constant with respect to the four-point definition of hyperbolicity; the inequalities for the hyperbolicity constant with respect to a definition can be translated to the hyperbolicity constant with respect to another definition, with additional multiplicative and/or additive constants (for instance, multiplying or dividing by 3 the initial upper or lower bound, respectively). But note that it is not difficult to check that circular-arc graphs are hyperbolic; for a fixed definition of hyperbolicity, the challenge is to obtain sharp bounds for the hyperbolicity constant.

\section{BACKGROUND AND PREVIOUS RESUltS}

We collect in this section some previous definitions and results which will be useful along the paper. 
We say that the curve $\gamma$ in a metric space $X$ is a geodesic if we have $L\left(\left.\gamma\right|_{[t, s]}\right)=d(\gamma(t), \gamma(s))=|t-s|$ for every $s, t \in[a, b]$ (then $\gamma$ is equipped with an arc-length parametrization). The metric space $X$ is said geodesic if for every couple of points in $X$ there exists a geodesic joining them; we denote by $[x y]$ any geodesic joining $x$ and $y$; this notation is ambiguous, since in general we do not have uniqueness of geodesics, but it is very convenient. Consequently, any geodesic metric space is connected. If the metric space $X$ is a graph, then the edge joining the vertices $u$ and $v$ will be denoted by $u v$.

Throughout this paper, $G=(V, E)=(V(G), E(G))$ denotes a (finite or infinite) simple (without loops and multiple edges) graph (not necessarily connected) such that $V \neq \emptyset$ and every edge has length 1 . In order to consider a graph $G$ as a geodesic metric space, identify (by an isometry) any edge $u v \in E(G)$ with the interval $[0,1]$ in the real line; then the edge $u v$ (considered as a graph with just one edge) is isometric to the interval $[0,1]$. Thus, the points in $G$ are the vertices and, also, the points in the interior of any edge of $G$. In this way, any connected graph $G$ has a natural distance defined on its points, induced by taking shortest paths in $G$, and we can see $G$ as a metric graph. We denote by $d_{G}$ or $d$ this distance. If $x, y$ are in different connected components of $G$, we define $d_{G}(x, y)=\infty$. These properties guarantee that any connected component of any graph is a geodesic metric space.

If $X$ is a geodesic metric space and $x_{1}, x_{2}, x_{3} \in X$, the union of three geodesics $\left[x_{1} x_{2}\right],\left[x_{2} x_{3}\right]$ and $\left[x_{3} x_{1}\right]$ is a geodesic triangle that will be denoted by $T=\left\{x_{1}, x_{2}, x_{3}\right\}$ and we will say that $x_{1}, x_{2}$ and $x_{3}$ are the vertices of $T$; it is usual to write also $T=\left\{\left[x_{1} x_{2}\right],\left[x_{2} x_{3}\right],\left[x_{3} x_{1}\right]\right\}$. We say that $T$ is $\delta$-thin if any side of $T$ is contained in the $\delta$-neighborhood of the union of the two other sides. We denote by $\delta(T)$ the sharp thin constant of $T$, i.e., $\delta(T):=\inf \{\delta \geq 0 \mid T$ is $\delta$-thin $\}$. The space $X$ is $\delta$-hyperbolic (or satisfies the Rips condition with constant $\delta$ ) if every geodesic triangle in $X$ is $\delta$-thin. If we have a triangle with two identical vertices, we call it a "bigon". Obviously, every bigon in a $\delta$-hyperbolic space is $\delta$-thin. We denote by $\delta(X)$ the sharp hyperbolicity constant of $X$, i.e., $\delta(X):=\sup \{\delta(T) \mid T$ is a geodesic triangle in $X\}$. We say that $X$ is hyperbolic if $X$ is $\delta$-hyperbolic for some $\delta \geq 0$; then $X$ is hyperbolic if and only if $\delta(X)<\infty$. If $X$ has connected components $\left\{X_{i}\right\}_{i \in I}$, then we define $\delta(X):=\sup _{i \in I} \delta\left(X_{i}\right)$, and we say that $X$ is hyperbolic if $\delta(X)<\infty$.

In the classical references on this subject (see, e.g., [22]) appear several different definitions of Gromov hyperbolicity, which are equivalent in the sense that if $X$ is $\delta$-hyperbolic with respect to one definition, then it is $\delta^{\prime}$-hyperbolic with respect to another definition (for some $\delta^{\prime}$ related to $\delta$ ). We have chosen this definition by its deep geometric meaning 22 .

We want to remark that the main examples of hyperbolic graphs are the trees. In fact, the hyperbolicity constant of a geodesic metric space can be viewed as a measure of how "tree-like" the space is, since those spaces $X$ with $\delta(X)=0$ are precisely the metric trees. This is an interesting subject since, in many applications, one finds that the borderline between tractable and intractable cases may be the tree-like degree of the structure to be dealt with (see, e.g., 14]). However, the hyperbolicity constant does not relate the graph in question to a specific tree (if connected) or forest (if not connected). In [45, a measure called forest likelihood is introduced to estimate the likelihood of any given forest via a random dynamical generation process. This measure establishes an interesting connection between static graphs and dynamically growing graphs.

For any graph $G$, we define, as usual,

$$
\begin{gathered}
\operatorname{diam} V(G):=\sup \left\{d_{G}(v, w) \mid v, w \in V(G)\right\}, \\
\operatorname{diam} G:=\sup \left\{d_{G}(x, y) \mid x, y \in G\right\} .
\end{gathered}
$$

i.e, $\operatorname{diam} V(G)$ is the diameter of the set of vertices of $G$, and $\operatorname{diam} G$ is the diameter of the whole graph $G$ (recall that in order to have a geodesic metric space, $G$ must contain both the vertices and the points in the interior of any edge of $G$ ).

The following result is well-known (see, e.g., [41, Theorem 8] for a proof).

Lemma 2.1. In any graph $G$ the inequality $\delta(G) \leq \frac{1}{2} \operatorname{diam} G$ holds. 
We say that a subgraph $\Gamma$ of $G$ is isometric if $d_{\Gamma}(x, y)=d_{G}(x, y)$ for every $x, y \in \Gamma$.

We need the following elementary results (see, e.g., [11, Lemma 5 and Theorem 11] for some proofs).

Lemma 2.2. If $\Gamma$ is an isometric subgraph of $G$, then $\delta(\Gamma) \leq \delta(G)$.

Lemma 2.3. If $C_{n}$ denotes the cycle graph with $n \geq 3$ vertices, then $\delta\left(C_{n}\right)=n / 4$.

If $G$ is a circular-arc graph, then a set of vertices $K=\left\{v_{1}, \ldots, v_{r}\right\}$ and corresponding $\operatorname{arcs}\left\{I_{1}, \ldots, I_{r}\right\}$ is said total if $I_{1} \cup \cdots \cup I_{r}=S S$, and we say that $r$ is the size of $K$.

We say that a circular-arc graph $G$ is $N I$ if it has a total set of vertices. If either $G$ is a finite circular-arc graph or every arc is open, then $G$ is $N I$ if and only if the union of the corresponding arcs to vertices in $G$ is $S S$. Note that a circular-arc graph $G$ is also an interval graph if and only if it is not NI. In 40] the authors study the hyperbolicity constant of interval graphs.

For any $N I$ circular-arc graph $G$, let us define

$$
\varrho(G):=\min \{\operatorname{size}(K) \mid K \text { is a total set of vertices in } G\} .
$$

If $G$ is an interval graph, then we define $\varrho(G):=0$. Hence, a circular-arc graph $G$ is NI if and only if $\varrho(G) \geq 1$. Note that $\varrho=1$ if and only if an arc is the whole unit circle $S S$.

As usual, by cycle we mean a simple closed curve, i.e., a path with different vertices, unless the last one, which is equal to the first vertex.

Given a graph $G$, we denote by $J(G)$ the union of the set $V(G)$ and the midpoints of the edges of $G$. Consider the set $\mathbb{T}_{1}$ of geodesic triangles $T$ in $G$ that are cycles and such that the three vertices of the triangle $T$ belong to $J(G)$, and denote by $\delta_{1}(G)$ the infimum of the constants $\lambda$ such that every triangle in $\mathbb{T}_{1}$ is $\lambda$-thin.

The following result, which appears in [4, Theorems 2.5, 2.6 and 2.7], will be used throughout the paper.

Theorem 2.4. For every graph $G$ we have $\delta_{1}(G)=\delta(G)$. Furthermore, if $G$ is hyperbolic, then $\delta(G)$ is a multiple of $1 / 4$ and there exists $T \in \mathbb{T}_{1}$ with $\delta(T)=\delta(G)$.

The following result in [35, Theorem 11] will be useful.

Theorem 2.5. If $G$ is a graph with edges of length 1 with $\delta(G)<1$, then we have either $\delta(G)=0$ or $\delta(G)=3 / 4$. Furthermore,

- $\delta(G)=0$ if and only if $G$ is a tree.

- $\delta(G)=3 / 4$ if and only if $G$ is not a tree and every cycle in $G$ has length 3 .

We say that a vertex $v$ in a graph $G$ is a cut-vertex if $G \backslash v$ is not connected. A graph is biconnected if it does not contain cut-vertices. Given a graph $G$, we say that a family of subgraphs $\left\{G_{s}\right\}_{s}$ of $G$ is a $T$-decomposition of $G$ if $\cup_{s} G_{s}=G$ and $G_{s} \cap G_{r}$ is either a cut-vertex or the empty set for each $s \neq r$. The well-known biconnected decomposition of any graph is an example of T-decomposition.

It is known that the hyperbolicity constant of a graph is the supremum of the hyperbolicity constants of its biconnected components 23 . One can check that the following result also holds (see, e.g., 5, Theorem 3] for a proof).

Proposition 2.6. Let $G$ be a graph and $\left\{G_{s}\right\}_{s}$ be any $T$-decomposition of $G$, then

$$
\delta(G)=\sup _{s} \delta\left(G_{s}\right) .
$$

By [35, Proposition 5 and Theorem 7], we have the following result.

Lemma 2.7. If $G$ is any graph with a cycle $g$ with length $L(g) \geq 3$, then $\delta(G) \geq 3 / 4$. If there exists a cycle $g$ in $G$ with length $L(g) \geq 4$, then $\delta(G) \geq 1$. 
We recall some facts from [40].

Let $G$ be an interval graph.

We say that $G$ has the 0-intersection property if for every three corresponding intervals $I^{\prime}, I^{\prime \prime}$ and $I^{\prime \prime \prime}$ to vertices in $G$ we have $I^{\prime} \cap I^{\prime \prime} \cap I^{\prime \prime \prime}=\emptyset$.

$G$ has the (3/4)-intersection property if it does not have the 0-intersection property and for every four corresponding intervals $I^{\prime}, I^{\prime \prime}, I^{\prime \prime \prime}$ and $I^{\prime \prime \prime \prime \prime}$ to vertices in $G$ we have $I^{\prime} \cap I^{\prime \prime} \cap I^{\prime \prime \prime}=\emptyset$ or $I^{\prime} \cap I^{\prime \prime} \cap I^{\prime \prime \prime \prime}=\emptyset$.

By a couple of intervals in a cycle $C$ of $G$ we mean the union of two non-disjoint intervals whose corresponding vertices belong to $C$. We say that $G$ has the 1-intersection property if it does not have the 0 and (3/4)-intersection properties and for every cycle $C$ in $G$ each interval and couple of corresponding intervals to vertices in $C$ are not disjoint.

Let $G$ be an interval graph. We say that $G$ has the (3/2)-intersection property if there exists two disjoint corresponding intervals $I^{\prime}$ and $I^{\prime \prime}$ to vertices in a cycle $C$ in $G$ such that there is no interval $I$ (corresponding to a vertex in $G$ ) with $I \cap I^{\prime} \neq \emptyset$ and $I \cap I^{\prime \prime} \neq \emptyset$.

[40, Theorem 3.14] give the following result.

Theorem 2.8. Every interval graph $G$ is hyperbolic and $\delta(G) \in\{0,3 / 4,1,5 / 4,3 / 2\}$. Furthermore,

- $\delta(G)=0$ if and only if $G$ has the 0-intersection property.

- $\delta(G)=3 / 4$ if and only if $G$ has the (3/4)-intersection property.

- $\delta(G)=1$ if and only if $G$ has the 1-intersection property.

- $\delta(G)=5 / 4$ if and only if $G$ does not have the $0,3 / 4,1$ and (3/2)-intersection properties.

- $\delta(G)=3 / 2$ if and only if $G$ has the (3/2)-intersection property.

It is well-known that the interval graphs $G$ with $\delta(G)=0$ are the caterpillar trees (the trees for which removing the leaves and incident edges produces a path graph), see [27, but the characterization with the 0 -intersection property is interesting, since it looks similar to the other intersection properties.

If $C$ is a cycle in $G$ and $v \in V(G)$, we denote by $\operatorname{deg}_{C}(v)$ the degree of the vertex $v$ in the subgraph $\Gamma$ induced by $V(C)$ (note that $\Gamma$ could contain edges that are not contained in $C$, and thus it is possible to have $\operatorname{deg}_{C}(v)>2$ ).

In [3, Theorem 3.2] appears the following result.

Theorem 2.9. Given any graph $G$, we have $\delta(G) \geq 5 / 4$ if and only if there exist a cycle $g$ in $G$ with length $L(g) \geq 5$ and a vertex $w \in g$ such that $\operatorname{deg}_{g}(w)=2$.

The following result appears in [25, Theorem 4.9].

Theorem 2.10. If $G$ is a graph with $n$ vertices and minimum degree $n-3$, then $\delta(G) \leq 5 / 4$.

The following result in [6. Theorem 2.2] gives a sharp bound for the hyperbolicity constant of the complement of a graph.

Theorem 2.11. If $G$ is a graph with $\operatorname{diam}(V(G)) \geq 3$, then its complement graph $\bar{G}$ satisfies $0 \leq \delta(\bar{G}) \leq 2$.

Finally, we will need the following result in [40, Theorem 4.8] that improves Theorem 2.11 for interval graphs (recall that the most difficult case in the study of the complement of a graph is the set of graphs $G$ with $\operatorname{diam} V(G)=2)$.

Theorem 2.12. If $G$ is an interval graph, then $0 \leq \delta(\bar{G}) \leq 2$.

\section{CIRCUlAR-ARC GRAPHS AND HYPERBOLICITY}

The parameter $\varrho(G)$ plays an important role in the study of the hyperbolicity of circular-arc graphs, as the following result shows. Recall that $\lfloor t\rfloor$ denotes the lower integer part of the real number $t$, i.e., the greatest integer least than or equal to $t$.

Since any NI circular-arc graph is a bounded set, we have that it is hyperbolic. The following result provides sharp inequalities for the hyperbolicity constant of any circular-arc graph. 
Theorem 3.1. Let $G$ be a circular-arc graph. If $\varrho(G) \neq 1,2$, then $G$ satisfies the sharp inequalities

$$
\frac{1}{4} \varrho(G) \leq \delta(G) \leq \frac{1}{2}\left\lfloor\frac{1}{2} \varrho(G)\right\rfloor+\frac{3}{2} .
$$

If $\varrho(G)=1$, then $G$ satisfies the sharp inequalities

$$
0 \leq \delta(G) \leq \frac{3}{2}
$$

If $\varrho(G)=2$, then $G$ satisfies the sharp inequalities

$$
0 \leq \delta(G) \leq 2 .
$$

Proof. The result is known if $G$ is an interval graph (i.e., if $\varrho(G)=0$ ), see [40, Corollary 4.1].

Assume now that $\varrho(G) \geq 1$. Let us prove the upper bound of $\delta(G)$. Fix any set of vertices $K=$ $\left\{v_{1}, \ldots, v_{\varrho(G)}\right\}$ and corresponding $\operatorname{arcs}\left\{I_{1}, \ldots, I_{\varrho(G)}\right\}$ with $I_{1} \cup \cdots \cup I_{\varrho(G)}=S S$. Thus, every arc in $S S$ intersects some $\operatorname{arc}$ in $\left\{I_{1}, \ldots, I_{\varrho(G)}\right\}$. Hence,

$$
\begin{aligned}
\operatorname{diam} V(G) & \leq 1+\operatorname{diam} K+1=\left\lfloor\frac{1}{2} \varrho(G)\right\rfloor+2, \\
\operatorname{diam} G & \leq \frac{1}{2}+\operatorname{diam} V(G)+\frac{1}{2} \leq\left\lfloor\frac{1}{2} \varrho(G)\right\rfloor+3,
\end{aligned}
$$

and Lemma 2.1 gives the upper bound.

Let us prove now that this bound is sharp. Given $\theta_{1}<\theta_{2}$, denote by $\left[e^{i \theta_{1}}, e^{i \theta_{2}}\right]$ the arc

$$
\left[e^{i \theta_{1}}, e^{i \theta_{2}}\right]:=\left\{e^{i \theta} \mid \theta \in\left[\theta_{1}, \theta_{2}\right]\right\} .
$$

Fix any even integer $\varrho \geq 6$ with $\varrho \equiv 2(\bmod 4)$ and consider the family of arcs

$$
\left\{\left[e^{2 \pi i(j-1) / \varrho}, e^{2 \pi i j / \varrho}\right]\right\}_{j=1}^{\varrho}
$$

Denote by $I_{j}$ the $\operatorname{arc}\left[e^{2 \pi i(j-1) / \varrho}, e^{2 \pi i j / \varrho}\right]$. Let $z_{1}, z_{2}, z_{3}$ be the points $e^{\pi i /(2 \varrho)}, e^{2 \pi i /(2 \varrho)}, e^{3 \pi i /(2 \varrho)}$ in $I_{1}$, respectively, $z_{4}, z_{5}$ the points $e^{2 \pi i / \varrho+2 \pi i /(3 \varrho)}, e^{2 \pi i / \varrho+4 \pi i /(3 \varrho)}$ in $I_{2}$, respectively, and $z_{j}$ the midpoint of $I_{j-3}$ with $6 \leq j \leq \varrho / 2+3$. Let $z_{\varrho / 2+4}, z_{\varrho / 2+5}, z_{\varrho / 2+6}$ be the points $-e^{\pi i /(2 \varrho)},-e^{2 \pi i /(2 \varrho)},-e^{3 \pi i /(2 \varrho)}$ in $I_{\varrho / 2+1}$, respectively, $z_{\varrho / 2+7}, z_{\varrho / 2+8}$ the points $-e^{2 \pi i / \varrho+2 \pi i /(3 \varrho)},-e^{2 \pi i / \varrho+4 \pi i /(3 \varrho)}$ in $I_{\varrho / 2+2}$, respectively, and $z_{k}$ the midpoint of $I_{k-6}$ with $\varrho / 2+9 \leq k \leq \varrho+6$.

Consider the circular-arc graph $G_{\varrho}$ defined as the intersection graph of the family of arcs

$$
\left\{\left[e^{2 \pi i(j-1) / \varrho}, e^{2 \pi i j / \varrho}\right]\right\}_{j=1}^{\varrho} \cup\left\{\left[z_{j}, z_{j+1}\right]\right\}_{j=1}^{\varrho+6} \cup\left\{\left[z_{\varrho+6}, z_{1}\right]\right\} .
$$

Let $x$ (respectively, $y$ ) be the midpoint of the edge of $G_{\varrho}$ with endpoints corresponding to the arcs $\left[z_{1}, z_{2}\right]$ and $\left[z_{2}, z_{3}\right]$ (respectively, $\left[z_{\varrho / 2+4}, z_{\varrho / 2+5}\right]$ and $\left[z_{\varrho / 2+5}, z_{\varrho / 2+6}\right]$ ).

We have $d_{G_{\varrho}}(x, y)=3 / 2+\varrho / 2+3 / 2=\varrho / 2+3$. Let $\gamma_{1}$ and $\gamma_{2}$ be two geodesics in $G_{\varrho}$ joining $x$ and $y$ such that $\gamma_{1}$ contains the corresponding vertices to the $\operatorname{arcs}\left\{\left[z_{j}, z_{j+1}\right]\right\}_{j=2}^{\varrho / 2+4}$ and $\gamma_{2}$ contains the corresponding vertices to the arcs $\left\{\left[z_{j}, z_{j+1}\right]\right\}_{j=\varrho / 2+5}^{\varrho+6} \cup\left\{\left[z_{\varrho+6}, z_{1}\right]\right\} \cup\left\{\left[z_{1}, z_{2}\right]\right\}$. Consider the geodesic bigon $\left\{\gamma_{1}, \gamma_{2}\right\}$. If $p$ is the midpoint of $\gamma_{1}$, then $d_{G_{\varrho}}\left(p, \gamma_{2}\right)=d_{G_{\varrho}}(x, y) / 2=\varrho / 4+3 / 2$. Hence, $\varrho / 4+3 / 2=d_{G_{\varrho}}\left(p, \gamma_{2}\right) \leq \delta\left(G_{\varrho}\right) \leq$ $\varrho / 4+3 / 2$, and we conclude $\delta\left(G_{\varrho}\right)=\varrho / 4+3 / 2$.

If $\varrho(G)=1$, then this upper bound is also attained. The wheel graph with seven vertices $W_{7}$ is a circular-arc graph with $\varrho(G)=1$, and [41, Theorem 11] gives that $\delta\left(W_{7}\right)=3 / 2$.

If $\varrho(G)=2$, then this upper bound is attained by the circular-arc graph $G$ corresponding to the arcs

$$
\begin{aligned}
{\left[e^{0 i}, e^{\pi i}\right] \cup\left[e^{\pi i}, e^{2 \pi i}\right] } & \cup\left[e^{0 i}, e^{\pi i / 4}\right] \cup\left[e^{\pi i / 4}, e^{\pi i / 2}\right] \cup\left[e^{\pi i / 2}, e^{3 \pi i / 4}\right] \cup\left[e^{3 \pi i / 4}, e^{\pi i}\right] \\
& \cup\left[e^{\pi i}, e^{5 \pi i / 4}\right] \cup\left[e^{5 \pi i / 4}, e^{3 \pi i / 2}\right] \cup\left[e^{3 \pi i / 2}, e^{7 \pi i / 4}\right] \cup\left[e^{7 \pi i / 4}, e^{2 \pi i}\right] .
\end{aligned}
$$

In order to prove $\delta(G)=2$, let $x$ (respectively, $y$ ) be the midpoint of the edge in $G$ with endpoints $\left[e^{\pi i / 4}, e^{\pi i / 2}\right]$ and $\left[e^{\pi i / 2}, e^{3 \pi i / 4}\right]$ (respectively, $\left[e^{5 \pi i / 4}, e^{3 \pi i / 2}\right]$ and $\left[e^{3 \pi i / 2}, e^{7 \pi i / 4}\right]$ ). One can check that there are two geodesics 
$\gamma_{1}$ and $\gamma_{2}$ such that the midpoint $p$ of $\gamma_{1}$ satisfies $d_{G}\left(p, \gamma_{2}\right)=2$. If we consider the geodesic bigon $\left\{\gamma_{1}, \gamma_{2}\right\}$, then $2=d_{G}\left(p, \gamma_{2}\right) \leq \delta(G) \leq 2$, and we conclude $\delta(G)=2$.

In order to prove the lower bound of $\delta(G)$, we deal first with the case $\varrho(G) \geq 3$. As above, fix any set of vertices $K=\left\{v_{1}, \ldots, v_{\varrho(G)}\right\}$ and corresponding $\operatorname{arcs}\left\{I_{1}, \ldots, I_{\varrho(G)}\right\}$ with $I_{1} \cup \cdots \cup I_{\varrho(G)}=S S$. The definition of $\varrho(G)$ gives that the subgraph $\Gamma_{K}$ of $G$ induced by $K$ is an isometric subgraph of $G$. Since $\varrho(G) \geq 3$, the subgraph $\Gamma_{K}$ is isomorphic to the cycle graph $C_{\varrho(G)}$. Therefore, Lemmas 2.2 and 2.3 give $\delta(G) \geq \delta\left(\Gamma_{K}\right)=\varrho(G) / 4$.

Any circular-arc graph isomorphic to the cycle graph $C_{\varrho}$ attains this lower bound.

Finally, the lower bounds for the cases $\varrho(G)=1,2$ are trivial, and they are attained by the graphs $G_{1}$ with just a vertex and $G_{2}$ with just an edge, respectively.

An important subset of circular-arc graphs are proper circular-arc graphs. A circular-arc graph $G$ is said proper if there is a representation of $G$ where none of the arcs contains another. The following result improves Theorem 3.1 for this kind of graphs.

Theorem 3.2. Let $G$ be a proper circular-arc graph. If $\varrho(G) \geq 3$, then $G$ satisfies the sharp inequalities

$$
\frac{1}{4} \varrho(G) \leq \delta(G) \leq \frac{1}{2}\left\lfloor\frac{1}{2} \varrho(G)\right\rfloor+1 .
$$

If $\varrho(G)=1$, then $\delta(G)=0$. If $\varrho(G)=2$, then $G$ satisfies the sharp inequalities $0 \leq \delta(G) \leq 5 / 4$.

Proof. Assume first that $\varrho(G)=1$. Since $S S$ is a corresponding arc to a vertex of $G$ and $G$ is a proper circular-arc graph, we have that it has just a vertex, and $\delta(G)=0$.

Assume now that $\varrho(G) \geq 2$. The lower bounds are a consequence of Theorem 3.1 (note that the examples in the proof of Theorem 3.1 attaining the lower bounds are proper circular-arc graphs). Let us prove the upper bound of $\delta(G)$. Fix any set of vertices $K=\left\{v_{1}, \ldots, v_{\varrho(G)}\right\}$ and corresponding $\operatorname{arcs}\left\{I_{1}, \ldots, I_{\varrho(G)}\right\}$ with $I_{1} \cup \cdots \cup I_{\varrho(G)}=S S$. Thus, every arc in $S S$ intersects two $\operatorname{arcs}$ in $\left\{I_{1}, \ldots, I_{\varrho(G)}\right\}$. Given any $u, w \in V(G) \backslash K$, there are $v_{1, u}, v_{2, u}, v_{1, w}, v_{2, w} \in K$ with $u v_{1, u}, u v_{2, u}, w v_{1, w}, w v_{2, w}, v_{1, u} v_{2, u}, v_{1, w} v_{2, w} \in E(G)$. Thus,

$$
d_{G}\left(v_{1, u} v_{2, u}, v_{1, w} v_{2, w}\right) \leq\left\lfloor\frac{1}{2}(\varrho(G)-2)\right\rfloor=\left\lfloor\frac{1}{2} \varrho(G)\right\rfloor-1 .
$$

Hence,

$$
\begin{aligned}
\operatorname{diam} V(G) & \leq 1+\left(\left\lfloor\frac{1}{2} \varrho(G)\right\rfloor-1\right)+1=\left\lfloor\frac{1}{2} \varrho(G)\right\rfloor+1, \\
\operatorname{diam} G & \leq \frac{1}{2}+\operatorname{diam} V(G)+\frac{1}{2} \leq\left\lfloor\frac{1}{2} \varrho(G)\right\rfloor+2,
\end{aligned}
$$

and Lemma 2.1 gives the upper bound for $\varrho(G) \geq 3$, and $\delta(G) \leq 3 / 2$ if $\varrho(G)=2$.

Let us prove now that the upper bound for $\varrho(G) \geq 3$ is sharp. Fix any even integer $\varrho \geq 4$ and $0<\varepsilon<\pi / \varrho$, and consider the proper circular-arc graph $\Gamma_{\varrho}$ defined as the intersection graph of the family of arcs

$$
\begin{aligned}
\left\{\left[e^{2 \pi i(j-1) / \varrho}, e^{2 \pi i j / \varrho}\right]\right\}_{j=1}^{\varrho} & \cup\left\{\left[e^{\varepsilon i+2 \pi i(j-1) / \varrho}, e^{\varepsilon i+2 \pi i j / \varrho}\right]\right\}_{j=1}^{\varrho / 2-1} \cup\left\{\left[e^{\varepsilon i+\pi i+2 \pi i(j-1) / \varrho}, e^{\varepsilon i+\pi i+2 \pi i j / \varrho}\right]\right\}_{j=1}^{\varrho / 2-1} \\
& \cup\left[e^{-\varepsilon i+\pi i-2 \pi i / \varrho}, e^{-\varepsilon i+\pi i}\right] \cup\left[e^{-\varepsilon i+2 \pi i-2 \pi i / \varrho}, e^{-\varepsilon i+2 \pi i}\right] \\
& \cup\left[e^{-\varepsilon i / 2}, e^{\varepsilon i}\right] \cup\left[e^{-\varepsilon i / 2+\pi i}, e^{\varepsilon i+\pi i}\right] \\
& \cup\left[e^{-\varepsilon i+\pi i}, e^{\varepsilon i / 2+\pi i}\right] \cup\left[e^{-\varepsilon i+2 \pi i}, e^{\varepsilon i / 2+2 \pi i}\right] .
\end{aligned}
$$

Let $x$ (respectively, $y$ ) be the midpoint of the edge of $\Gamma_{\varrho}$ with endpoints corresponding to the $\operatorname{arcs}\left[e^{-\varepsilon i / 2}, e^{\varepsilon i}\right]$ and $\left[e^{-\varepsilon i+2 \pi i}, e^{\varepsilon i / 2+2 \pi i}\right]$ (respectively, $\left[e^{-\varepsilon i / 2+\pi i}, e^{\varepsilon i+\pi i}\right]$ and $\left[e^{-\varepsilon i+\pi i}, e^{\varepsilon i / 2+\pi i}\right]$ ). We have $d_{\Gamma_{\varrho}}(x, y)=\varrho / 2+2$. Let $\gamma_{1}$ and $\gamma_{2}$ be two geodesics in $\Gamma_{\varrho}$ joining $x$ and $y$ such that $\gamma_{1}$ contains the corresponding vertices to the $\operatorname{arcs}$

$$
\left[e^{-\varepsilon i / 2}, e^{\varepsilon i}\right] \cup\left\{\left[e^{\varepsilon i+2 \pi i(j-1) / \varrho}, e^{\varepsilon i+2 \pi i j / \varrho}\right]\right\}_{j=1}^{\varrho / 2-1} \cup\left[e^{-\varepsilon i+\pi i-2 \pi i / \varrho}, e^{-\varepsilon i+\pi i}\right] \cup\left[e^{-\varepsilon i+\pi i}, e^{\varepsilon i / 2+\pi i}\right]
$$


and $\gamma_{2}$ contains the corresponding vertices to the arcs

$\left[e^{-\varepsilon i / 2+\pi i}, e^{\varepsilon i+\pi i}\right] \cup\left\{\left[e^{\varepsilon i+\pi i+2 \pi i(j-1) / \varrho}, e^{\varepsilon i+\pi i+2 \pi i j / \varrho}\right]\right\}_{j=1}^{\varrho / 2-1} \cup\left[e^{-\varepsilon i+2 \pi i-2 \pi i / \varrho}, e^{-\varepsilon i+2 \pi i}\right] \cup\left[e^{-\varepsilon i+2 \pi i}, e^{\varepsilon i / 2+2 \pi i}\right]$.

Consider the geodesic bigon $\left\{\gamma_{1}, \gamma_{2}\right\}$. If $p$ is the midpoint of $\gamma_{1}$, then $d_{\Gamma_{\varrho}}\left(p, \gamma_{2}\right)=d_{\Gamma_{\varrho}}(x, y) / 2=\varrho / 4+1$. Hence, $\varrho / 4+1=d_{\Gamma_{\varrho}}\left(p, \gamma_{2}\right) \leq \delta\left(\Gamma_{\varrho}\right) \leq \varrho / 4+1$, and we conclude $\delta\left(\Gamma_{\varrho}\right)=\varrho / 4+1$.

Assume that $\varrho(G)=2$. We have proved $\delta(G) \leq 3 / 2$. Seeking for a contradiction assume that $\delta(G)=3 / 2$. By Theorem 2.4, there exist $T=\{x, y, z\} \in \mathbb{T}_{1}$ and $p \in[x y]$ with $d_{G}(p,[x z] \cup[y z])=\delta(G)=3 / 2$. Since we have proved $\operatorname{diam} V(G) \leq 2$ and $\operatorname{diam} G \leq 3$, we have $d_{G}(x, y)=3, d_{G}(p,\{x, y\})=d_{G}(p,[x z] \cup[y z])=3 / 2$, $x, y \in J(G) \backslash V(G)$ and $p \in V(G)$. Hence, $x$ (respectively, $y$ ) is the midpoint of $u_{1} u_{2} \in E(G)$ with $u_{1}, u_{2} \in$ $V(G) \backslash K$ and corresponding arcs $H_{1}, H_{2}$ (respectively, the midpoint of $w_{1} w_{2} \in E(G)$ with $w_{1}, w_{2} \in V(G) \backslash K$ and corresponding arcs $\left.J_{1}, J_{2}\right)$. Note that each arc $H_{1}, H_{2}, J_{1}, J_{2}$ intersects $I_{1} \cap I_{2}$ and it is different from $I_{1}$ and $I_{2}$. Since $G$ is a proper circular-arc graph, we have that both $H_{1}$ and $H_{2}$ contain the same connected component $\Lambda$ of $I_{1} \cap I_{2}$; also, both $J_{1}$ and $J_{2}$ contain the other connected component $\Lambda^{\prime}$ of $I_{1} \cap I_{2}$. Denote by $I$ the corresponding arc to $p$. Since $G$ is a proper circular-arc graph, we have that $I$ contains either $\Lambda$ or $\Lambda^{\prime}$. Assume that $I$ contains $\Lambda$ (if $I$ contains $\Lambda^{\prime}$, then the argument is similar). Thus, $d_{G}\left(p, u_{1}\right)=d_{G}\left(p, u_{2}\right)=1$. Without loss of generality we can assume that $u_{1} \in[x y]$. Therefore, $u_{2} \in[x z] \cup[y z]$ and we conclude $3 / 2=d_{G}(p,[x z] \cup[y z]) \leq d_{G}\left(p, u_{2}\right)=1$, a contradiction. Hence, $\delta(G)<3 / 2$ and Theorem 2.4 gives $\delta(G) \leq 5 / 4$.

Finally, we show that the proper circular-arc graph $\Gamma$ (with $\varrho(G)=2$ ) corresponding to the arcs

$$
\left[e^{0 i}, e^{\pi i}\right] \cup\left[e^{\pi i}, e^{2 \pi i}\right] \cup\left[e^{-\pi i / 8}, e^{\pi i / 4}\right] \cup\left[e^{-\pi i / 4}, e^{\pi i / 8}\right] \cup\left[e^{\pi i-\pi i / 8}, e^{\pi i+\pi i / 4}\right] \cup\left[e^{\pi i-\pi i / 4}, e^{\pi i+\pi i / 8}\right],
$$

satisfies $\delta(\Gamma)=5 / 4$. Let $x$ (respectively, $y$ ) be the midpoint of the edge in $\Gamma$ with endpoints $\left[e^{-\pi i / 8}, e^{\pi i / 4}\right]$ and $\left[e^{-\pi i / 4}, e^{\pi i / 8}\right]$ (respectively, $\left[e^{\pi i-\pi i / 8}, e^{\pi i+\pi i / 4}\right]$ and $\left[e^{\pi i-\pi i / 4}, e^{\pi i+\pi i / 8}\right]$ ). We have $d_{\Gamma}(x, y)=3$. One can check that there are two geodesics $\gamma_{1}$ and $\gamma_{2}$ such that the midpoint $q$ of $\gamma_{1}$ is a vertex of $\Gamma$ and $d_{\Gamma}\left(q, \gamma_{2}\right)=1$. If $p$ is a point in $\gamma_{1}$ with $d_{\Gamma}(p, q)=1 / 4$, then $d_{\Gamma}\left(p, \gamma_{2}\right)=5 / 4$. If we consider the geodesic bigon $\left\{\gamma_{1}, \gamma_{2}\right\}$, then $5 / 4=d_{\Gamma}\left(p, \gamma_{2}\right) \leq \delta(\Gamma) \leq 5 / 4$, and we conclude $\delta(\Gamma)=5 / 4$.

Note that Theorem 3.5 below gives a sufficient condition in order to attain the lower bound of $\delta(G)$ in Theorem 3.1. This sufficient condition is, in fact, a characterization when $3 \leq \varrho(G) \leq 4$.

Next, we are going to characterize the circular-arc graphs with the two smallest possible values for the hyperbolicity constant: 0 and $3 / 4$.

We say that a circular-arc graph $G$ has the 0-property if we have either:

(1) $G$ is an interval graph with the 0 -intersection property.

(2) $\varrho(G)=1$ and given two corresponding arcs $I, J$ to vertices in $G$ with $I, J \neq S S$, we have $I \cap J=\emptyset$.

(3) $\varrho(G)=2$ and there exist two corresponding $\operatorname{arcs} I_{1}, I_{2}$ to vertices in $G$ with $I_{1} \cup I_{2}=S S$ such any other corresponding arc to some vertex in $G$ intersects just one of the $\operatorname{arcs} I_{1}, I_{2}$, and if $G_{j}$ is the interval graph corresponding to the arcs intersecting $I_{j}$ then $G_{j}$ has the 0 -intersection property for $j=1,2$.

Proposition 3.3. A circular-arc graph $G$ satisfies $\delta(G)=0$ if and only if $G$ has the 0-property.

Proof. If $G$ is an interval graph, then Theorem 2.8 gives the result. Assume now that $G$ is a NI circular-arc graph.

If $G$ satisfies (2) in the definition of 0-property, then $G$ is a tree (in fact, it is a star graph) and we have $\delta(G)=0$.

If $G$ satisfies (3) in the definition of 0-property, then $G$ is a tree and we have $\delta(G)=0$.

Assume that $\delta(G)=0$. Theorem 3.1 gives that $\varrho(G) \leq 2$.

Assume that $\varrho(G)=1$. Seeking for a contradiction assume that there exist two corresponding arcs $I, J$ to vertices in $G$ with $I, J \neq S S$ and $I \cap J \neq \emptyset$. Therefore, there exists a cycle with length three corresponding to the $\operatorname{arcs} I, J, S S$, and Lemma 2.7 gives $0=\delta(G) \geq 3 / 4$, a contradiction. Thus, we have $I \cap J=\emptyset$ and $G$ has the 0-property. 
Assume that $\varrho(G)=2$. Thus, there exist two corresponding arcs $I_{1}, I_{2}$ to vertices in $G$ with $I_{1} \cup I_{2}=S S$. Seeking for a contradiction assume that there exists a corresponding arc $I$ to some vertex in $G$ intersecting both $\operatorname{arcs} I_{1}$ and $I_{2}$. Therefore, there is a cycle of length 3 in $G$ corresponding to $I, I_{1}, I_{2}$, and we have $\delta(G) \geq 3 / 4$ by Lemma 2.7, which is a contradiction. So, any other corresponding arc to some vertex in $G$ intersects just one of the $\operatorname{arcs} I_{1}, I_{2}$. Let $G_{j}$ be the interval graph corresponding to the arcs intersecting $I_{j}$ for $j=1,2$. Since $\delta(G)=0$, Proposition 2.6 gives that $\delta\left(G_{1}\right)=\delta\left(G_{2}\right)=0$. Thus, Theorem 2.8 gives that $G_{1}$ and $G_{2}$ have the 0-intersection property.

We say that a circular-arc graph $G$ has the (3/4)-property if we have either:

(1) $G$ is an interval graph with the (3/4)-intersection property.

(2) $\varrho(G)=1$, there exist two corresponding arcs $I^{\prime}, I^{\prime \prime} \neq S S$ to vertices in $G$ with $I^{\prime} \cap I^{\prime \prime} \neq \emptyset$, and for every three corresponding $\operatorname{arcs} I, J, K \neq S S$ to vertices in $G$, we have either $I \cap J=\emptyset$ or $I \cap K=\emptyset$.

(3) $\varrho(G)=2$ and there exist two corresponding arcs $I_{1}, I_{2}$ to vertices in $G$ with $I_{1} \cup I_{2}=S S$ such any other corresponding arc to some vertex in $G$ intersects just one of the $\operatorname{arcs} I_{1}, I_{2}$, and if $G_{j}$ is the interval graph corresponding to the arcs intersecting $I_{j}$ then $G_{1}$ has the (3/4)-intersection property and $G_{2}$ has either the 0 - or the $(3 / 4)$-intersection property.

(4) $\varrho(G)=2$ and there exist three corresponding arcs $I, I_{1}, I_{2}$ to vertices in $G$ with $I_{1} \cup I_{2}=S S$ and $I \cap I_{j} \neq \emptyset$ for $j=1,2$, such that any other arc corresponding to some vertex in $G$ intersects just one of the $\operatorname{arcs} I_{1}, I_{2}$ and does not intersect $I$, and if $G_{j}$ is the interval graph corresponding to the arcs intersecting $I_{j}$ except for $I$ then $G_{j}$ has either the 0 - or the (3/4)-intersection property for each $j=1,2$.

(5) $\varrho(G)=3$ and there exist three corresponding $\operatorname{arcs} I_{1}, I_{2}, I_{3}$ to vertices in $G$ with $I_{1} \cup I_{2} \cup I_{3}=S S$ such any other corresponding arc to some vertex in $G$ intersects just one of the $\operatorname{arcs} I_{1}, I_{2}, I_{3}$, and if $G_{j}$ is the interval graph corresponding to the arcs intersecting $I_{j}$ then $G_{j}$ has either the 0 - or the (3/4)-intersection property for each $j=1,2,3$.

Proposition 3.4. A circular-arc graph $G$ satisfies $\delta(G)=3 / 4$ if and only if $G$ has the (3/4)-property.

Proof. If $G$ is an interval graph, then Theorem 2.8 gives the result. Assume now that $G$ is a NI circular-arc graph.

If $G$ satisfies either (2), (3), (4) or (5) in the definition of (3/4)-property, then Theorems 2.5 and 2.8 and Proposition 2.6 give that $\delta(G)=3 / 4$.

Assume that $\delta(G)=3 / 4$. Theorem 3.1 gives $\varrho(G) \leq 3$.

Assume that $\varrho(G)=3$. Thus, there exist three corresponding $\operatorname{arcs} I_{1}, I_{2}, I_{3}$ to vertices in $G$ with $I_{1} \cup$ $I_{2} \cup I_{3}=S S$ Seeking for a contradiction assume that there exists a corresponding arc $I$ to some vertex in $G$ intersecting at least two arcs in $\left\{I_{1}, I_{2}, I_{3}\right\}$. Therefore, there is a cycle of length 4 in $G$ corresponding to $I, I_{1}, I_{2}, I_{3}$ and we have $\delta(G) \geq 1$ by Lemma 2.7, which is a contradiction. So, any other corresponding arc to some vertex in $G$ intersects just one of the $\operatorname{arcs} I_{1}, I_{2}, I_{3}$. Let $G_{j}$ be the interval graph corresponding to the arcs intersecting $I_{j}$ for $j=1,2,3$. Let us denote by $G_{0}$ the subgraph of $G$ induced by the corresponding vertices to $I_{1}, I_{2}, I_{3}\left(G_{0}\right.$ is a cycle graph with three vertices). Note that $\left\{G_{0}, G_{1}, G_{2}, G_{3}\right\}$ is a T-decomposition of $G$. Since $\delta(G)=3 / 4$, Proposition 2.6 gives that $\delta\left(G_{j}\right) \leq 3 / 4$ for $j=1,2,3$. Thus, Theorem 2.8 gives that $G_{j}$ has either the 0 - or the (3/4)-intersection property for each $j=1,2,3$, and we obtain condition (5).

Assume that $\varrho(G)=2$. Thus, there exist two corresponding arcs $I_{1}, I_{2}$ to vertices in $G$ with $I_{1} \cup I_{2}=S S$.

Assume that any other corresponding arc to some vertex in $G$ intersects just one of the $\operatorname{arcs} I_{1}, I_{2}$. Let $G_{j}$ be the interval graph corresponding to the $\operatorname{arcs}$ intersecting $I_{j}$ for $j=1,2$, and let $G_{0}$ be the subgraph of $G$ induced by the corresponding vertices to $I_{1}, I_{2}\left(G_{0}\right.$ has just an edge). Since $\left\{G_{0}, G_{1}, G_{2}\right\}$ is a T-decomposition of $G$, Proposition 2.6 gives

$$
\frac{3}{4}=\delta(G)=\max \left\{\delta\left(G_{0}\right), \delta\left(G_{1}\right), \delta\left(G_{2}\right)\right\}=\max \left\{\delta\left(G_{1}\right), \delta\left(G_{2}\right)\right\} .
$$

Hence, a subgraph, say $G_{1}$, has hyperbolicity constant $3 / 4$ and $\delta\left(G_{2}\right) \leq 3 / 4$. Thus, Theorems 2.5 and 2.8 give that $G_{1}$ has the (3/4)-intersection property and $G_{2}$ has either the 0 - or the (3/4)-intersection property, and we obtain condition (3). 
Assume that there exist corresponding arcs $I, I_{1}, I_{2}$ to vertices in $G$ with $I \cap I_{j} \neq \emptyset$ for $j=1,2$. Seeking for a contradiction assume that there exists another corresponding arc $J$ to some vertex in $G$ intersecting both $\operatorname{arcs} I_{1}$ and $I_{2}$. Hence, there is a cycle of length four in $G$ corresponding to $I, I_{1}, I_{2}, J$ and we have $\delta(G) \geq 1$ by Lemma 2.7, which is a contradiction. Thus, any other corresponding arc to some vertex in $G$ intersects just one of the arcs $I_{1}, I_{2}$. A similar argument gives that any other corresponding arc to some vertex in $G$ does not intersect $I$. Let $G_{j}$ be the interval graph corresponding to the arcs intersecting $I_{j}$ except for $I$. Let us denote by $G_{0}$ the subgraph of $G$ induced by the corresponding vertices to $I, I_{1}, I_{2}\left(G_{0}\right.$ is a cycle graph with three vertices). Since $\left\{G_{0}, G_{1}, G_{2}\right\}$ is a T-decomposition of $G$, Proposition 2.6 gives

$$
\frac{3}{4}=\delta(G)=\max \left\{\delta\left(G_{0}\right), \delta\left(G_{1}\right), \delta\left(G_{2}\right)\right\}=\max \left\{\frac{3}{4}, \delta\left(G_{1}\right), \delta\left(G_{2}\right)\right\} .
$$

This equation holds if and only if $\delta\left(G_{j}\right) \leq 3 / 4$ for $j=1,2$. Thus, Theorems 2.5 and 2.8 give that $G_{j}$ has either the 0 - or the (3/4)-intersection property for each $j=1,2$, and we obtain condition (4).

Finally, assume that $\varrho(G)=1$.

Seeking for a contradiction assume that for every two corresponding $\operatorname{arcs} I^{\prime}, I^{\prime \prime} \neq S S$ to vertices in $G$, we have $I^{\prime} \cap I^{\prime \prime}=\emptyset$. Thus, $G$ is a star graph and $\delta(G)=0$, a contradiction. Hence, there exist two arcs $I^{\prime}, I^{\prime \prime} \neq S S$ with $I^{\prime} \cap I^{\prime \prime} \neq \emptyset$.

Seeking for a contradiction assume that there exist three corresponding $\operatorname{arcs} I, J, K \neq S S$ to vertices in $G$ with $I \cap J \neq \emptyset$ and $I \cap K \neq \emptyset$. Therefore, there exists a cycle with length four corresponding to the arcs $I, J, K, S S$, and Lemma 2.7 gives $\delta(G) \geq 1$, a contradiction. Thus, for every three $\operatorname{arcs} I, J, K \neq S S$ we have either $I \cap J=\emptyset$ or $I \cap K=\emptyset$, and we obtain condition (2).

We say that a circular-arc graph $G$ with $\varrho(G) \geq 3$ has the $\varrho(G)$-property if there exist $\varrho(G)$ corresponding $\operatorname{arcs} I_{1}, \ldots, I_{\varrho(G)}$ to vertices in $G$ with $I_{1} \cup \cdots \cup I_{\varrho(G)}=S S$ such any other corresponding arc to some vertex in $G$ intersects just one of the $\operatorname{arcs} I_{1}, \ldots, I_{\varrho(G)}$, and if $G_{j}$ is the interval graph corresponding to the $\operatorname{arcs}$ intersecting $I_{j}$ for $1 \leq j \leq \varrho(G)$ and $\varrho(G) \leq 5$ then:

(1) $G_{j}$ has either the 0 - or (3/4)-intersection property for $1 \leq j \leq \varrho(G)$ if $\varrho(G)=3$.

(2) $G_{j}$ has either the 0-, (3/4)- or 1-intersection property for $1 \leq j \leq \varrho(G)$ if $\varrho(G)=4$.

(3) $G_{j}$ does not have the (3/2)-intersection property for $1 \leq j \leq \varrho(G)$ if $\varrho(G)=5$.

The next result gives a sufficient condition in order to attain the lower bound of $\delta(G)$ in Theorem 3.1 We also prove that this sufficient condition is, in fact, a characterization when $3 \leq \varrho(G) \leq 4$.

Theorem 3.5. Let $G$ be a circular-arc graph with $\varrho(G) \geq 3$. If $G$ has the $\varrho(G)$-property, then $G$ satisfies $\delta(G)=\varrho(G) / 4$. Furthermore, if $\delta(G)=\varrho(G) / 4$ with $3 \leq \varrho(G) \leq 4$, then $G$ has the $\varrho(G)$-property.

Proof. Assume first that $G$ has the $\varrho(G)$-property. Let us denote by $G_{0}$ the subgraph of $G$ induced by the corresponding vertices to $I_{1}, \ldots, I_{\varrho(G)}\left(G_{0}\right.$ is a cycle graph with $\varrho(G)$ vertices). Since $\left\{G_{0}, G_{1}, \ldots, G_{\varrho(G)}\right\}$ is a T-decomposition of $G$, Theorem 3.1, Proposition 2.6 and Lemma 2.3 give

$$
\frac{\varrho(G)}{4} \leq \delta(G)=\max \left\{\delta\left(G_{0}\right), \delta\left(G_{1}\right), \ldots, \delta\left(G_{\varrho(G)}\right)\right\}=\max \left\{\frac{\varrho(G)}{4}, \delta\left(G_{1}\right), \ldots, \delta\left(G_{\varrho(G)}\right)\right\} .
$$

Since $G_{j}$ is an interval graph for $1 \leq j \leq \varrho(G)$, if $\varrho(G) \geq 6$, then Theorem 2.8 gives $\delta\left(G_{j}\right) \leq 3 / 2 \leq \varrho(G) / 4$ for $1 \leq j \leq \varrho(G)$.

If $\varrho(G)=3$, then Theorem 2.8 gives $\delta\left(G_{j}\right) \leq 3 / 4=\varrho(G) / 4$ for $1 \leq j \leq \varrho(G)$.

If $\varrho(G)=4$, then Theorem 2.8 gives $\delta\left(G_{j}\right) \leq 1=\varrho(G) / 4$ for $1 \leq j \leq \varrho(G)$.

If $\varrho(G)=5$, then Theorem 2.8 gives $\delta\left(G_{j}\right) \leq 5 / 4=\varrho(G) / 4$ for $1 \leq j \leq \varrho(G)$.

These inequalities and (3.1) give $\delta(G)=\varrho(G) / 4$ in every case.

Assume now that $\delta(G)=\varrho(G) / 4$ with $3 \leq \varrho(G) \leq 4$.

Seeking for a contradiction assume that there exists a corresponding arc $I$ to some vertex in $G$ intersecting at least two arcs in $\left\{I_{1}, \ldots, I_{\varrho(G)}\right\}$. Denote by $\left\{v_{1}, \ldots, v_{\varrho(G)}\right\}$ their corresponding vertices in $G$, and by $C$ the cycle in $G$ with vertices $\left\{v_{1}, \ldots, v_{\varrho(G)}\right\}$. Let $v_{I}$ be the corresponding vertex in $G$ to $I$. 
If $\varrho(G)=3$, then there is a cycle in $G$ with vertices $\left\{v_{1}, v_{2}, v_{3}, v_{I}\right\}$, and Lemma 2.7 gives $\delta(G) \geq 1$, a contradiction.

If $\varrho(G)=4$, then we show now that there is a cycle in $G$ with vertices $\left\{v_{1}, v_{2}, v_{3}, v_{4}, v_{I}\right\}$. The definition of $\varrho(G)$ gives that $v_{I}$ is neighbor of at most three vertices in $\left\{v_{1}, v_{2}, v_{3}, v_{4}\right\}$. Without loss of generality we can assume that $v_{1} v_{2}, v_{2} v_{3}, v_{3} v_{4}, v_{4} v_{1}, v_{1} v_{I}, v_{2} v_{I} \in E(G)$ and $v_{4} v_{I} \notin E(G)$. Consider the cycle $g:=$ $v_{1} v_{I} \cup v_{I} v_{2} \cup v_{2} v_{3} \cup v_{3} v_{4} \cup v_{4} v_{1}$ in $G$. Since $L(g)=5$ and $\operatorname{deg}_{g}\left(v_{4}\right)=2$, Theorem 2.9 gives $\delta(G) \geq 5 / 4$, a contradiction.

Thus, any corresponding arc to some vertex in $G$ intersects just one of the $\operatorname{arcs}\left\{I_{1}, \ldots, I_{\varrho(G)}\right\}$. Let $G_{j}$ be the interval graph corresponding to the arcs intersecting $I_{j}$ for $1 \leq j \leq \varrho(G)$. Let us denote by $G_{0}$ the subgraph of $G$ induced by the corresponding vertices to $I_{1}, \ldots, I_{\varrho(G)}$ for $1 \leq j \leq \varrho(G)\left(G_{0}\right.$ is a cycle graph with $\varrho(G)$ vertices). Since $\left\{G_{0}, G_{1}, \ldots, G_{\varrho(G)}\right\}$ is a T-decomposition of $G$, Proposition 2.6 and Lemma 2.3 give

$$
\frac{\varrho(G)}{4}=\delta(G)=\max \left\{\delta\left(G_{0}\right), \delta\left(G_{1}\right), \ldots, \delta\left(G_{\varrho(G)}\right)\right\}=\max \left\{\frac{\varrho(G)}{4}, \delta\left(G_{1}\right), \ldots, \delta\left(G_{\varrho(G)}\right)\right\} .
$$

This equation holds if and only if $\delta\left(G_{j}\right) \leq \varrho(G) / 4$ for $1 \leq j \leq \varrho(G)$.

If $\varrho(G)=3$, then Theorem 2.8 gives $\delta\left(G_{j}\right) \leq 3 / 4=\varrho(G) / 4$ for $1 \leq j \leq \varrho(G)$ if and only if (1) holds.

If $\varrho(G)=4$, then Theorem 2.8 gives $\delta\left(G_{j}\right) \leq 1=\varrho(G) / 4$ for $1 \leq j \leq \varrho(G)$ if and only if (2) holds.

Hence, $G$ has the $\varrho(G)$-property.

Example 3.6. The second statement in Theorem 3.5 does not hold for $\varrho \geq 5$, as the following example shows. Consider the graph $G_{\varrho}$ obtained from the cycle graph $C_{\varrho}$ with $\varrho$ vertices and an additional vertex $v_{I}$ connected by an edge with just three consecutive vertices in $C_{\varrho}$. We have that $G_{\varrho}$ is a circular-arc graph without the @-property. [35. Theorem 30] gives that the hyperbolicity constant of any graph with $n$ vertices is at most $n / 4$. Hence, $\varrho / 4 \leq \delta\left(G_{\varrho}\right) \leq(\varrho+1) / 4$. By using the characterization in [35, Theorem 30] of the graphs with $n$ vertices and hyperbolicity constant $n / 4$, we obtain $\delta\left(G_{\varrho}\right)<(\varrho+1) / 4$. Since $\delta(G)$ is a multiple of $1 / 4$ by Theorem 2.4, we conclude $\delta\left(G_{\varrho}\right)=\varrho / 4$.

\section{Complement And Line GRAPH}

In this section we obtain bounds for the hyperbolicity constant of the complement and line of a circular-arc graph, respectively. These theorems improve, for circular-arc graphs, the general bounds for the hyperbolicity constant of the complement and line graphs.

Given any graph $G$, we denote by $\bar{G}$ the complement of $G$, i.e., $\bar{G}$ is the graph with $V(\bar{G})=V(G)$ and $v w \in E(\bar{G})$ if and only if $v w \notin E(G)$.

Now, we are interested in the hyperbolicity of the complement of circular-arc graphs. Let us start with two technical results.

Lemma 4.1. Let $G$ be a circular-arc graph with $\varrho(G) \geq 1$. If two vertices $u$ and $v$ are not neighbors and have two common neighbors $v_{1}, v_{2}$, such that $v_{1}$ and $v_{2}$ are not neighbors, then their corresponding arcs satisfy $I_{u} \cup I_{v} \cup I_{v_{1}} \cup I_{v_{2}}=S S$.

Proof. Since $\varrho(G) \geq 1, I_{u} \cap I_{v}=\emptyset, I_{v_{1}} \cap I_{v_{2}}=\emptyset, I_{u} \cap I_{v_{j}} \neq \emptyset$ and $I_{v} \cap I_{v_{j}} \neq \emptyset$ for $j=1$, 2, we have $I_{u} \cup I_{v} \cup I_{v_{1}} \cup I_{v_{2}}=S S$.

Lemma 4.2. Let $G$ be a circular-arc graph with $\varrho(G)>4$. If two vertices $u$ and $v$ have two common neighbors $v_{1}, v_{2}$, such that $v_{1}$ and $v_{2}$ are not neighbors, then $u$ and $v$ are neighbors.

Proof. Seeking for a contradiction, assume that $u$ and $v$ are not neighbors. Lemma4.1 gives $I_{u} \cup I_{v} \cup I_{v_{1}} \cup I_{v_{2}}=$ $S S$, and thus $\varrho(G) \leq 4$, a contradiction. So, $u$ and $v$ are neighbors.

Recall that a graph is $s$-regular if every vertex has degree $s$, i.e., has $s$ neighbors. In order to prove Theorem 4.5 below we need the following surprising result about regular graphs which is interesting by itself. 
Theorem 4.3. Let $G$ be a $(n-3)$-regular graph with $n \geq 5$ vertices. Then $\delta(G)=1$ if $\bar{G}$ is a union of cycle graphs with three vertices, and $\delta(G)=5 / 4$ otherwise.

Proof. Assume first that $\bar{G}$ is a union of cycle graphs with three vertices (thus, $n \geq 6$ ). [24, Lemma 5.7] gives that $\delta(G) \leq 1$. Since $n \geq 6$, we have $n-3 \geq n / 2$ and there exists a Hamiltonian cycle with $n \geq 6$ vertices; thus, Lemma 2.7 gives that $\delta(G) \geq 1$.

Assume now that $\bar{G}$ is not a union of cycle graphs with three vertices. If $n=5$, then $G$ is a cycle graph with five vertices and $\delta(G)=5 / 4$. Assume that $n \geq 6$. Hence, there exists $v \in V(G)$ such that the connected component of $\bar{G}$ containing $v$ is not a cycle graph with three vertices. Let $v_{1}, v_{2}$ be the vertices with $v_{1} v, v_{2} v \notin E(G)$. Seeking for a contradiction assume that $v_{1} v_{2} \notin E(G)$. Thus, the connected component of $\bar{G}$ containing $v$ is the cycle graph with vertices $v, v_{1}, v_{2}$, a contradiction. Hence, $v_{1} v_{2} \in E(G)$. Since $n \geq 6$, we have $2(n-3) \geq n$ and there are at least two common neighbors of $v$ and $v_{j}$ for each $j=1,2$. Therefore, there exist two different vertices $v_{3}, v_{4}$ with $v_{3} v, v_{4} v, v_{3} v_{1}, v_{4} v_{2} \in E(G)$, and we have the cycle $g$ given by $v, v_{3}, v_{1}, v_{2}, v_{4}, v$ in $G$. Since $L(g)=5$ and $\operatorname{deg}_{g}(v)=2$ (recall that $v_{1} v, v_{2} v \notin E(G)$ ), Theorem 2.9 gives $\delta(G) \geq 5 / 4$. Finally, Theorem 2.10 gives $\delta(G) \leq 5 / 4$.

Theorem 4.3 has the following direct consequence.

Corollary 4.4. If $G$ is a $(n-3)$-regular graph with $n \geq 5$ vertices and $n$ is not a multiple of 3 , then $\delta(G)=5 / 4$.

The following result provides sharp bounds for the hyperbolicity constant of the complement of any circular-arc graph (even the circular-arc graphs $G$ with $\operatorname{diam} V(G)=2$ ). Note that it improves Theorem 2.11 for circular-arc graphs; recall that the most difficult case in the study of the complement of a graph are the graphs $G$ with diam $V(G)=2$ (this is the case if $\varrho(G)=4$ or $\varrho(G)=5$ ), and that Theorem 2.11 does not deal with these graphs.

Theorem 4.5. Let $G$ be a circular-arc graph. If $\varrho(G)=0$, then $0 \leq \delta(\bar{G}) \leq 2$. If $\varrho(G)>4$, then $5 / 4 \leq \delta(\bar{G}) \leq 3 / 2$. If $\varrho(G)=4$, then $0 \leq \delta(\bar{G}) \leq 7 / 2$. Furthermore, the lower bounds are sharp; in particular, they are attained by the cycle graphs for $\varrho(G) \geq 4$.

Proof. If $\varrho(G)=0$, then Theorem 2.12 gives the result.

Assume now that $\varrho(G)>4$. We are going to prove that $\operatorname{diam} \bar{G} \leq 3$ (note that it is possible to have $\operatorname{diam} V(G)=2$, and that the inequality $\operatorname{diam} \bar{G} \leq 3$ is stronger than $\operatorname{diam} V(\bar{G}) \leq 3$ ). Seeking for a contradiction assume that $\operatorname{diam} \bar{G}>3$.

Assume first that $\operatorname{diam} V(\bar{G}) \geq 4$. Thus, there exist $v, w \in V(\bar{G})$ with $d_{\bar{G}}(v, w)=4$. Let $v_{0}=$ $v, v_{1}, v_{2}, v_{3}, v_{4}=w \in V(\bar{G})$ such that $v_{j-1} v_{j} \in E(\bar{G})$ for $1 \leq j \leq 4$. Therefore, $v_{0}$ and $v_{1}$ have two common neighbors $v_{3}, v_{4}$ in $G$ with $v_{3} v_{4} \notin E(G)$, and Lemma 4.2 gives that $v_{0}$ and $v_{1}$ are neighbors in $G$. This contradicts $v_{0} v_{1} \in E(\bar{G})$.

Assume that $\operatorname{diam} V(\bar{G})=3$. Thus, there exist $v \in V(\bar{G})$ and a midpoint $x$ of an edge $v_{3} v_{3}^{\prime}$ in $\bar{G}$ with $d_{\bar{G}}(v, x)=7 / 2$. Hence, there exist $v_{0}=v, v_{1}, v_{2} \in V(\bar{G})$ such that $v_{j-1} v_{j} \in E(\bar{G})$ for $1 \leq j \leq 3$. Therefore, $v_{3}$ and $v_{3}^{\prime}$ have two common neighbors $v_{0}, v_{1}$ in $G$ with $v_{0} v_{1} \notin E(G)$, and Lemma 4.2 gives that $v_{3}$ and $v_{3}^{\prime}$ are neighbors in $G$. This contradicts $v_{3} v_{3}^{\prime} \in E(\bar{G})$.

Hence, $\operatorname{diam} \bar{G} \leq 3$ and Lemma 2.1 gives $\delta(\bar{G}) \leq 3 / 2$.

In order to prove the lower bound, consider a cycle $C$ in $G$ given by $v_{1}, v_{2}, \ldots, v_{\varrho(G)}, v_{1}$ such that the subgraph induced by this vertices is $C$. Consider the cycle $g$ in $\bar{G}$ given by $v_{3}, v_{5}, v_{2}, v_{4}, v_{1}, v_{3}$. Since $L(g)=5$ and $\operatorname{deg}_{g}\left(v_{3}\right)=2\left(\right.$ recall that $v_{3} v_{2}, v_{3} v_{4} \notin E(\bar{G})$ ), Theorem 2.9 gives $\delta(\bar{G}) \geq 5 / 4$.

Consider now the cycle graph with $\varrho$ vertices $C_{\varrho}$. Since $\varrho>4, \bar{C}_{\varrho}$ is $(\varrho-3)$-regular and its complement is $C_{\varrho}$, Theorem 4.3 gives $\delta\left(\bar{C}_{\varrho}\right)=5 / 4$ and the bound is attained.

Finally, assume that $\varrho(G)=4$ and consider a geodesic triangle $T=\{x, y, z\}$ in $\bar{G}$ and $p \in[x y]$. By Theorem [2.4, we can assume that $x, y, z \in J(\bar{G})$. If $d_{\bar{G}}(x, y) \leq 4$, then $d_{\bar{G}}(p,[x z] \cup[y z]) \leq 2<7 / 2$. Assume that $d_{\bar{G}}(x, y)>4$. Since $x, y, z \in J(\bar{G})$, we have $d_{\bar{G}}(x, y) \geq 9 / 2$. Thus, there exist $u, v \in V(\bar{G}) \cap[x y]$ with 
$d_{\bar{G}}(u, v)=4$ and vertices $v_{0}=u, v_{1}, v_{2}, v_{3}, v_{4}=v \in V(\bar{G}) \cap[x y]$ such that $v_{j-1} v_{j} \in E(\bar{G})$ for $1 \leq j \leq 4$ and $d_{\bar{G}}\left(p, v_{2}\right) \leq 1 / 2$. Therefore, $v_{0}$ and $v_{1}$ have two common neighbors $v_{3}, v_{4}$ in $G$, and $v_{0} v_{1}, v_{3} v_{4} \notin E(G)$. Hence, Lemma 4.1 gives that their corresponding arcs satisfy $I_{v_{0}} \cup I_{v_{1}} \cup I_{v_{3}} \cup I_{v_{4}}=S S$. Seeking for a contradiction assume that there exists a vertex $w_{0} \in V(\bar{G})$ with corresponding $\operatorname{arc} I_{w_{0}}$ such that $I_{w_{0}} \cap I_{v_{j}} \neq \emptyset$ for $j=0,1,3,4$. Since $I_{v_{0}} \cup I_{v_{1}} \cup I_{v_{3}} \cup I_{v_{4}}=S S$, there exist $i, j \in\{0,1,3,4\}$ with $I_{w_{0}} \cup I_{v_{i}} \cup I_{v_{j}}=S S$. This contradicts $\varrho(G)=4$, and so every vertex $w_{0} \in V(\bar{G})$ has at most three neighbors in $\left\{v_{0}, v_{1}, v_{3} v_{4}\right\}$ in $G$. Thus, given any vertex $w_{0} \in V(\bar{G}) \cap([x z] \cup[y z])$, there exists $k \in\{0,1,3,4\}$ with $w_{0} v_{k} \notin E(G)$, and

$$
d_{\bar{G}}(p,[x z] \cup[y z]) \leq d_{\bar{G}}\left(p, w_{0}\right) \leq d_{\bar{G}}\left(p, v_{2}\right)+d_{\bar{G}}\left(v_{2}, v_{k}\right)+d_{\bar{G}}\left(v_{k}, w_{0}\right) \leq \frac{1}{2}+2+1=\frac{7}{2} .
$$

So, $\delta(\bar{G}) \leq 7 / 2$.

The lower bound $\delta(\bar{G}) \geq 0$ trivially holds. If we consider the cycle graph $G=C_{4}$, then $\bar{G}$ is the union of two disjoint edges and $\delta(\bar{G})=0$. Hence, the lower bound is attained.

Remark 4.6. Note that Theorems 2.4 and 4.5 give that if $G$ is a circular-arc graph with $\varrho(G)>4$, then we have either $\delta(\bar{G})=5 / 4$ or $\delta(\bar{G})=3 / 2$.

Theorems 3.1 and 4.5 have the following consequence.

Corollary 4.7. If $G$ is a circular-arc graph with $\varrho(G) \geq 7$, then $\delta(\bar{G})<\delta(G)$.

In 1956, Nordhaus and Gaddum gave lower and upper bounds on the sum and the product of the chromatic number of a graph and its complement in [36. Since then, relations of a similar type have been proposed for many other graph invariants, in several hundred papers (see, e.g., [1]).

Also, Theorems 3.1 and 4.5 provide some Nordhaus-Gaddum type results.

Corollary 4.8. If $G$ is a circular-arc graph, then

$$
\begin{aligned}
& \frac{5 \varrho(G)}{16} \leq \delta(G) \delta(\bar{G}) \leq \frac{3 \varrho(G)}{8}+\frac{9}{4}, \quad \frac{\varrho(G)+5}{4} \leq \delta(G)+\delta(\bar{G}) \leq \frac{\varrho(G)}{4}+3, \quad \text { if } \varrho(G)>4, \\
& 0 \leq \delta(G) \delta(\bar{G}) \leq \frac{7 \varrho(G)}{8}+\frac{21}{4}, \quad \frac{\varrho(G)}{4} \leq \delta(G)+\delta(\bar{G}) \leq \frac{\varrho(G)}{4}+5, \quad \text { if } \varrho(G)=4, \\
& 0 \leq \delta(G) \delta(\bar{G}) \leq \frac{\varrho(G)}{2}+3, \quad \frac{\varrho(G)}{4} \leq \delta(G)+\delta(\bar{G}) \leq \frac{\varrho(G)}{4}+\frac{7}{2}, \quad \text { if } \varrho(G)=0 .
\end{aligned}
$$

If $G$ is a graph with edges $E(G)=\left\{e_{i}\right\}_{i \in \mathcal{I}}$, the line graph $\mathrm{L}(G)$ of $G$ is a graph which has a vertex $v_{e_{i}} \in V(\mathrm{~L}(G))$ for each edge $e_{i}$ of $G$, and an edge joining $v_{e_{i}}$ and $v_{e_{j}}$ when $e_{i} \cap e_{j} \neq \emptyset$. The line graph of $G$ is interesting in the theory of geometric graphs, since it is the intersection graph of $E(G)$.

A graph is chordal if all cycles of length at least four have a chord, which is an edge that is not part of the cycle but connects two vertices of the cycle (i.e., it does not have induced cycles of length greater than three).

The following result appears in [9, Lemma 2.2].

Lemma 4.9. Suppose that $G$ is chordal, and that $x_{1}, x_{2}, \ldots, x_{n}, x_{1}$ is a cycle in $G$, where $n \geq 4$. If $d\left(x_{1}, x_{3}\right)=2$, then there exists $i \in\{4,5, \ldots, n\}$ such that $x_{i} x_{2} \in E(G)$.

We want to prove a similar result for $\mathrm{E}(G)$. In order to do it we need some background.

Given $v_{e} \in V(\mathrm{E}(G))$, let us define $h\left(v_{e}\right)$ as the midpoint of the edge $e \in E(G)$ and $H\left(v_{e}\right)=e$. Thus, $h$ and $H$ are maps with $h: V(\mathrm{E}(G)) \rightarrow G$ and $H: V(\mathrm{E}(G)) \rightarrow E(G)$.

[11, Remark 3.3] gives that the map $h$ is an isometry:

Lemma 4.10. For every $x, y \in V(L(G))$, we have

$$
d_{E(G)}(x, y)=d_{G}(h(x), h(y)) .
$$


Lemma 4.11. Suppose that $G$ is chordal, and that $u_{1}, u_{2}, \ldots, u_{n}, u_{1}$ is a cycle in $E(G)$, where $n \geq 6$. If $d_{E(G)}\left(u_{1}, u_{4}\right)=3$, then there exists $i \in\{4,5, \ldots, n\}$ and $u \in V(E(G))$ such that $u_{2} u, u_{3} u, u_{i} u, u_{i+1} u \in$ $E(L(G))$, where $u_{n+1}=u_{1}$.

Proof. Denote by $C$ the cycle $u_{1}, u_{2}, \ldots, u_{n}, u_{1}$ and by $C_{0}$ its corresponding cycle in $G$. Lemma 4.10 gives $d_{G}\left(h\left(u_{1}\right), h\left(u_{4}\right)\right)=d_{\mathrm{E}(G)}\left(u_{1}, u_{4}\right)=3$. Thus, the vertices $H\left(u_{1}\right) \cap H\left(u_{2}\right)$ and $H\left(u_{3}\right) \cap H\left(u_{4}\right)$ in $C_{0}$ satisfy $d_{G}\left(H\left(u_{1}\right) \cap H\left(u_{2}\right), H\left(u_{3}\right) \cap H\left(u_{4}\right)\right)=2$, and Lemma 4.9 gives that there exists $i \in\{4,5, \ldots, n\}$ with $d_{G}\left(H\left(u_{2}\right) \cap H\left(u_{3}\right), H\left(u_{i}\right) \cap H\left(u_{i+1}\right)\right)=1$. If we denote by $u$ the corresponding vertex $\operatorname{in} \mathrm{\textrm {L }}(G)$ to the edge in $G$ with endpoints $H\left(u_{2}\right) \cap H\left(u_{3}\right)$ and $H\left(u_{i}\right) \cap H\left(u_{i+1}\right)$, then $u_{2} u, u_{3} u, u_{i} u, u_{i+1} u \in E(\mathrm{E}(G))$.

The following result in [11, Corollary 3.12] relates the hyperbolicity constants of $G$ and $\mathrm{E}(G)$.

Theorem 4.12. For any graph $G$ we have

$$
\delta(G) \leq \delta(E(G)) \leq 5 \delta(G)+\frac{5}{2} .
$$

If we consider the four-point definition of hyperbolicity, another usual definition, and we denote by $\delta^{\prime}$ the sharp constant for this definition, we have the following result in [18, Theorem 6].

Theorem 4.13. For any graph $G$ we have

$$
\delta^{\prime}(G)-1 \leq \delta^{\prime}(E(G)) \leq \delta^{\prime}(G)+1 .
$$

The upper bound in Theorem 4.13 allows to improve the upper bound in Theorem 4.12 to $\delta(\mathrm{E}(G)) \leq$ $3 \delta(G)+c$ for some constant $c$ (unfortunately, the constant $c$ is greater than 1 since the four-point definition considers as points just the vertices of the graph, and it is necessary an additional constant in order to deal with continuous triangles).

In order to study the line of circular-arc graphs (see Theorem4.15 below) we need the following result about the line of chordal graphs which is interesting by itself, and improves the upper bound $\delta(\mathrm{E}(G)) \leq 5 \delta(G)+5 / 2$ in Theorem 4.12 (and even the bound $\delta(\mathrm{E}(G)) \leq 3 \delta(G)+c$ ) for chordal graphs (since $\delta(G) \leq 3 / 2$ for every chordal graph $G$, see [9]).

Theorem 4.14. If $G$ is a chordal graph, then

$$
\delta(L(G)) \leq \frac{5}{2} .
$$

Proof. Let us consider a geodesic triangle $T=\{x, y, z\}$ in $\mathrm{E}(G)$ and $p \in[x y]$. By Theorem 2.4, we can assume that $T$ is a cycle.

We are going to prove $d_{\mathrm{Ł}_{(G)}}(p,[x z] \cup[y z]) \leq 3$. Without loss of generality we can assume that $d_{\mathrm{Ł}_{(G)}}(p,[x z] \cup$ $[y z]) \geq 2$. If we denote the vertices of the cycle $T$ by $u_{1}, u_{2}, \ldots, u_{n}, u_{1}$, then we can assume that $p \in u_{2} u_{3}$. Since $d_{\mathrm{Ł}(G)}(p,\{x, y\}) \geq d_{\mathrm{Ł}(G)}(p,[x z] \cup[y z]) \geq 2$, we have $u_{1} u_{2} \cup u_{2} u_{3} \cup u_{3} u_{4} \subset[x y]$ and so, $d_{\mathrm{\complement}(G)}\left(u_{1}, u_{4}\right)=3$. By Lemma 4.11, there exist $j \in\{2,3\}, k \in\{4,5, \ldots, n\}$ and $u \in V(\mathrm{E}(G))$ such that $u_{2} u, u_{3} u, u_{k} u \in E(\mathrm{~L}(G))$ and $d_{T}\left(u_{j}, u_{k}\right) \geq 3$. Since $u_{j} \in[x y], d_{\mathrm{\complement}(G)}\left(u_{j}, u_{k}\right)=2$ and $d_{T}\left(u_{j}, u_{k}\right) \geq 3$, we have $u_{k} \in[x z] \cup[y z]$ and

$$
d_{\mathrm{\complement}(G)}(p,[x z] \cup[y z]) \leq d_{\mathrm{\complement}(G)}\left(p, u_{k}\right) \leq d_{\mathrm{Ł}_{(G)}}\left(p,\left\{u_{2}, u_{3}\right\}\right)+d_{\mathrm{\complement}_{(G)}}\left(\left\{u_{2}, u_{3}\right\}, u\right)+d_{\mathrm{\complement}(G)}\left(u, u_{k}\right) \leq \frac{5}{2} .
$$

Hence, $\delta(\mathrm{L}(G)) \leq 5 / 2$.

The following result improves the upper bound in Theorem 4.12 for circular-arc graphs.

Theorem 4.15. Let $G$ be a circular-arc graph. If $\varrho(G) \geq 3$, then

If $\varrho(G)=0,2$, then

$$
\frac{1}{4} \varrho(G) \leq \delta(E(G)) \leq \frac{1}{2}\left\lfloor\frac{1}{2} \varrho(G)\right\rfloor+\frac{5}{2} .
$$

$$
0 \leq \delta(L(G)) \leq \frac{5}{2}
$$


If $\varrho(G)=1$, then

$$
0 \leq \delta(E(G)) \leq 2 .
$$

Proof. Theorems 3.1 and 4.12 give the lower bounds.

If $\varrho(G)=0$, then Theorem 4.14 gives the upper bound, since every interval graph is chordal.

Assume that $\varrho(G)>0$ and let us prove the upper bounds of $\delta(\mathrm{E}(G))$. Fix any set of vertices $K=$ $\left\{v_{1}, \ldots, v_{\varrho(G)}\right\}$ and corresponding $\operatorname{arcs}\left\{I_{1}, \ldots, I_{\varrho(G)}\right\}$ with $I_{1} \cup \cdots \cup I_{\varrho(G)}=S S$.

Assume first $\varrho(G) \geq 3$, and denote by $C$ the cycle in $G$ with $V(C)=K$ and by $C^{\prime}$ the corresponding cycle in $\mathrm{E}(G)$ to $C$. Since every vertex in $G$ is at distance at most 1 from $C$, every vertex in $\mathrm{E}(G)$ is at distance at most 2 from $C^{\prime}$, and

$$
\begin{aligned}
\operatorname{diam} V(\mathrm{E}(G)) & \leq 2+\operatorname{diam} V\left(C^{\prime}\right)+2=\left\lfloor\frac{1}{2} \varrho(G)\right\rfloor+4, \\
\operatorname{diam} \mathrm{E}(G) & \leq\left\lfloor\frac{1}{2} \varrho(G)\right\rfloor+5,
\end{aligned}
$$

and Lemma 2.1 gives the upper bound.

If $\varrho(G)=2$, then the previous argument gives the desired upper bound, by taking $v_{v_{1} v_{2}}$ (with diameter zero) instead of $C^{\prime}$.

If $\varrho(G)=1$, then every vertex in $G$ is a neighbor of $v_{1}$ and the set of edges in $G$ incident on $v_{1}$ corresponds

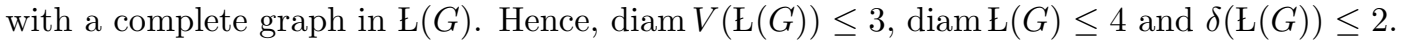

\section{REFERENCES}

[1] Aouchiche, M. and Hansen, P., A survey of Nordhaus-Gaddum type relations, Discrete Appl. Math. 161 (2013), $466-546$.

[2] Bermudo, S., Carballosa, W., Rodríguez, J. M. and Sigarreta, J. M., On the hyperbolicity of edge-chordal and path-chordal graphs, Filomat 30:9 (2016), 2599-2607.

[3] Bermudo, S., Rodríguez, J. M., Rosario, O. and Sigarreta, J. M., Small values of the hyperbolicity constant in graphs, Discrete Math. 339 (2016), 3073-3084.

[4] Bermudo, S., Rodríguez, J. M. and Sigarreta, J. M., Computing the hyperbolicity constant, Comput. Math. Appl. 62 (2011), 4592-4595.

[5] Bermudo, S., Rodríguez, J. M., Sigarreta, J. M. and Vilaire, J.-M., Gromov hyperbolic graphs, Discr. Math. 313 (2013), $1575-1585$.

[6] Bermudo, S., Rodríguez, J. M., Sigarreta, J. M. and Tourís, E., Hyperbolicity and complement of graphs, Appl. Math. Letters 24 (2011), 1882-1887.

[7] Boguñá, M., Papadopoulos, F. and Krioukov, D., Sustaining the Internet with Hyperbolic Mapping, Nature Commun. $\mathbf{1}(62)$ (2010), $18 \mathrm{p}$.

[8] Brandstädt, A., Chepoi, V. and Dragan, F., Distance Approximating Trees for Chordal and Dually Chordal Graphs, J. Algor. 30 (1999), 166-184.

[9] Brinkmann, G., Koolen J. and Moulton ,V., On the hyperbolicity of chordal graphs, Ann. Comb. 5 (2001), 61-69.

[10] Calegari, D. and Fujiwara, K., Counting subgraphs in hyperbolic graphs with symmetry, J. Math. Soc. Japan 67 (2015), $1213-1226$

[11] Carballosa, W., Rodríguez, J. M. and Sigarreta, J. M., New inequalities on the hyperbolity constant of line graphs, Ars Combin. 129 (2016), 367-386.

[12] Chalopin, J., Chepoi, V., Papasoglu, P. and Pecatte, T., Cop and robber game and hyperbolicity, SIAM J. Discr. Math. 28:4 (2015), 1987-2007.

[13] Charney, R., Artin groups of finite type are biautomatic, Math. Ann. 292 (1992), 671-683.

[14] Chen, B., Yau, S.-T. and Yeh, Y.-N., Graph homotopy and Graham homotopy, Discrete Math. 241 (2001), 153-170.

[15] Chepoi, V., Dragan, F. F., Estellon, B., Habib, M. and Vaxes Y., Notes on diameters, centers, and approximating trees of $\delta$-hyperbolic geodesic spaces and graphs, Electr. Notes Discr. Math. 31 (2008), 231-234.

[16] Cohen, N., Coudert, D. and Lancin, A., Algorithme exact et approché pour le calcul de l'hyperbolicité d'un graphe. In Yann Nisse, Nicolas et Rousseau, Franck et Busnel, editor, Pornic, France, pages 1-4, May 2013, pp. 1-4.

[17] Coudert, D. and Ducoffe, G., Recognition of $C_{4}$-Free and 1/2-Hyperbolic Graphs, SIAM J. Discrete Math. 28 (2014), 1601-1617.

[18] Coudert, D. and Ducoffe, G., On the hyperbolicity of bipartite graphs and intersection graphs, Discrete App. Math. 214 (2016), 187-195.

[19] Coudert, D. and Ducoffe, G., Data center interconnection networks are not hyperbolic, Theor. Computer Sci. 639 (2016), $72-90$. 
[20] Fournier, H., Ismail, A. and Vigneron, A., Computing the Gromov hyperbolicity of a discrete metric space, J. Inform. Proc. Letters 115 (2015), 576-579.

[21] Frigerio, R. and Sisto, A., Characterizing hyperbolic spaces and real trees, Geom. Dedicata 142 (2009), 139-149.

[22] Ghys, E. and de la Harpe, P., Sur les Groupes Hyperboliques d'après Mikhael Gromov. Progress in Mathematics 83, Birkhäuser Boston Inc., Boston, MA, 1990.

[23] Gromov, M., Hyperbolic groups, in "Essays in group theory". Edited by S. M. Gersten, M. S. R. I. Publ. 8. Springer, 1987, 75-263.

[24] Hernández, V., Pestana, D. and Rodríguez, J. M., Bounds on Gromov hyperbolicity constant, RACSAM 110(2) (2016), 321-342.

[25] Hernández, V., Pestana, D. and Rodríguez, J. M., On a classical theorem on the diameter and minimum degree of a graph, to appear in Acta Math. Sinica.

[26] Jonckheere, E. A., Contrôle du traffic sur les réseaux à géométrie hyperbolique-Vers une théorie géométrique de la sécurité l'acheminement de l'information, J. Europ. Syst. Autom. 8 (2002), 45-60.

[27] Jürgen, E., Extremal interval graphs, J. Graph Theory 17 (1993), 117-127.

[28] Koolen, J. H. and Moulton, V., Hyperbolic Bridged Graphs, Europ. J. Comb. 23 (2002), 683-699.

[29] Krauthgamer, R. and Lee, J. R., Algorithms on negatively curved spaces, FOCS 2006.

[30] Krioukov, D., Papadopoulos, F., Kitsak, M., Vahdat, A. and Boguñá, M., Hyperbolic geometry of complex networks, Physical Review E 82, 036106 (2010).

[31] Li, S. and Tucci, G. H., Traffic Congestion in Expanders, $(p, \delta)$-Hyperbolic Spaces and Product of Trees, Internet Math. 11:2 (2015), 134-142.

[32] Martínez-Pérez, A., Chordality properties and hyperbolicity on graphs, Electr. J. Comb. 23(3) (2016), P3.51.

[33] McConnell, R. M., Linear-time recognition of circular-arc graphs, Algorithmica 37 (2003) 93-147.

[34] McKee, T. A. and McMorris, F. R., Topics in Intersection Graph Theory, SIAM, 1999.

[35] Michel, J., Rodríguez, J. M., Sigarreta, J. M. and Villeta, M., Hyperbolicity and parameters of graphs, Ars Comb. 100 (2011), 43-63.

[36] Nordhaus, E. A. and Gaddum, J., On complementary graphs, Amer. Math. Monthly 63 (1956), 175-177.

[37] Oshika, K., Discrete groups, AMS Bookstore, 2002.

[38] Pal, M., Intersection Graphs: An Introduction, Ann. Pure Appl. Math. 4 (2013), 43-91.

[39] Papasoglu, P., An algorithm detecting hyperbolicity, in Geometric and computational perspectives on infinite groups, DIMACS - Series in Discrete Mathematics and Theoretical Computer Science Volume 25, AMS, 1996, pp.193-200.

[40] Reyes, R., Rodríguez, J. M., Sigarreta, J. M. and Villeta, M., Hyperbolicity of interval graphs, Submitted.

[41] Rodríguez, J. M., Sigarreta, J. M., Vilaire, J.-M. and Villeta, M., On the hyperbolicity constant in graphs, Discr. Math. 311 (2011), 211-219.

[42] Shang, Y., Lack of Gromov-hyperbolicity in colored random networks, Pan-American Math. J. 21(1) (2011), 27-36.

[43] Shang, Y., Lack of Gromov-hyperbolicity in small-world networks, Cent. Eur. J. Math. 10 (2012), 1152-1158.

[44] Shang, Y., Non-hyperbolicity of random graphs with given expected degrees, Stoch. Models 29 (2013), 451-462.

[45] Shang, Y., On the likelihood of forests, Physica A: Stat. Mech. Appl. 456 (2016) 157-166.

[46] Shavitt, Y. and Tankel, T., On internet embedding in hyperbolic spaces for overlay construction and distance estimation, IEEE/ACM Trans. Networking 16:1 (2008), 25-36.

[47] Sigarreta, J. M., Hyperbolicity in median graphs, Proc. Indian Acad. Sci. Math. Sci. 123 (2013), 455-467.

[48] Tourís, E., Graphs and Gromov hyperbolicity of non-constant negatively curved surfaces, J. Math. Anal. Appl. 380 (2011), 865-881.

[49] Verbeek, K. and Suri, S., Metric embeddings, hyperbolic space and social networks, Comput. Geom. 59 (2016), 1-12.

[50] Wu, Y. and Zhang, C., Chordality and hyperbolicity of a graph, Electr. J. Comb. 18 (2011), P43.

Facultad de Matemáticas, Universidad Autónoma de Guerrero, Carlos E. Adame No.54 Col. Garita, 39650 Acalpulco Gro., Mexico

E-mail address: khanclawn@hotmail.com

Departamento de Matemáticas, Universidad Carlos ili de Madrid, Avenida de la Universidad 30, 28911 Leganés, MADRID, SPAIN

E-mail address: jomaro@math.uc3m.es

Facultad de Matemáticas, Universidad Autónoma de Guerrero, Carlos E. Adame No.54 Col. Garita, 39650 Acalpulco Gro., Mexico

E-mail address: josemariasigarretaalmira@hotmail.com

Departamento de Estadística e Investigación Operativa iII, Facultad de Estudios Estadísticos, Universidad Complutense de Madrid, Av. Puerta de Hierro s/n., 28040 Madrid, Spain

E-mail address: mvilleta@estad.ucm.es 